University of Wollongong

Research Online

Faculty of Informatics - Papers (Archive)

Faculty of Engineering and Information

Sciences

$1-1-2007$

\title{
Continuum modelling for carbon and boron nitride nanostructures
}

James M. Hill

University of Wollongong, jhill@uow.edu.au

Ngamta Thamwattana

University of Wollongong,ngamta@uow.edu.au

Follow this and additional works at: https://ro.uow.edu.au/infopapers

Part of the Physical Sciences and Mathematics Commons

\section{Recommended Citation}

Hill, James M. and Thamwattana, Ngamta: Continuum modelling for carbon and boron nitride nanostructures 2007, 1-16.

https://ro.uow.edu.au/infopapers/1369

Research Online is the open access institutional repository for the University of Wollongong. For further information contact the UOW Library: research-pubs@uow.edu.au 


\title{
Continuum modelling for carbon and boron nitride nanostructures
}

\author{
Abstract \\ Continmuum based models are presented here for certain boron nitride and carbon nanostructures. \\ Keywords \\ Continuum, modelling, for, carbon, boron, nitride, nanostructures \\ Disciplines \\ Physical Sciences and Mathematics \\ Publication Details \\ Thamwattana, N. \& Hill, J. M. (2007). Continuum modelling for carbon and boron nitride nanostructures. \\ Journal of Physics: Condensed Matter, 19 (40), 1-16.
}




\title{
Continuum modelling for carbon and boron nitride nanostructures
}

\author{
Ngamta Thamwattana and James $M$ Hill \\ Nanomechanics Group, School of Mathematics and Applied Statistics, University of Wollongong, \\ Wollongong, NSW 2522, Australia
}

\begin{abstract}
Continuum based models are presented here for certain boron nitride and carbon nanostructures. In particular, certain fullerene interactions, $\mathrm{C}_{60}-\mathrm{C}_{60}, \mathrm{~B}_{36} \mathrm{~N}_{36}-$ $B_{36} \mathrm{~N}_{36}$ and $\mathrm{C}_{60}-\mathrm{B}_{36} \mathrm{~N}_{36}$, and fullerene-nanotube oscillator interactions, $\mathrm{C}_{60}-$ boron nitride nanotube, $\mathrm{C}_{60}$-carbon nanotube, $\mathrm{B}_{36} \mathrm{~N}_{36}$-boron nitride nanotube and $\mathrm{B}_{36} \mathrm{~N}_{36}$-carbon nanotube, are studied using the Lennard-Jones potential and the continuum approach, which assumes a uniform distribution of atoms on the surface of each molecule. Issues regarding the encapsulation of a fullerene into a nanotube are also addressed, including acceptance and suction energies of the fullerenes, preferred position of the fullerenes inside the nanotube and the gigahertz frequency oscillation of the inner molecule inside the outer nanotube. Our primary purpose here is to extend a number of established results for carbon to the boron nitride nanostructures.
\end{abstract}

\section{Introduction}

Spherical and cylindrical cage-like molecules, the so-called fullerenes and nanotubes, are of considerable interest due to their unique properties and their proposed applications in constructing novel nanodevices. While early attention has been given to carbon nanotubes and carbon fullerenes, such as $\mathrm{C}_{60}$ molecules, researchers are now considering many other types of nanotubes and fullerenes (see, for example, [1]), and these include boron nitride (BN) nanotubes and fullerenes. As with carbon nanotubes, BN nanotubes can also be thought of as a tube formed by rolling up a hexagonal sheet of boron nitride. From Ishigami et al [2], graphite and hexagonal BN (h-BN), which are the parent materials of carbon nanotubes and boron nitride nanotubes respectively, are of similar structures. They are both layered materials composed of layers of hexagonal lattices. While graphite has carbon atoms at all lattice sites, h-BN comprises altemating boron $\mathrm{B}$ and nitrogen $\mathrm{N}$ atoms located at lattice sites. Although their structures are similar, $\mathrm{BN}$ nanotubes posses quite different properties to those of carbon nanotubes. One unique property of $\mathrm{BN}$ nanotubes is a wide energy band gap of approximately $5 \mathrm{eV}$, which is independent of the wall number, the diameter and the tube chirality. As such, BN nanotubes have potential applications as insulating nanostructures for geometrically and 
electronically confining atomic, molecular or nano-crystalline species [3]. Further, Hirano et al [4] expect that for BN hollow cage nanostructures their various properties, such as chemical stability, semiconductor and wear resistance, will be useful for electronic devices, high heat resistance semiconductors and insulator lubricants.

One well known hybrid structure is referred to as a nanopeapod, for which the hollow carbon nanotube is the pod and the fullerenes $\mathrm{C}_{60}$ are the peas inside the pod. Nanopeapods have attracted much attention, since they posses potential applications as superconducting nanowires $[5,6]$. The superiority of using nanopeapods instead of an empty carbon nanotube to create a superconducting nanowire is that the charge can travel not only along the tube wall, but also along the chain of fullerenes inside the nanotube. However, other studies of nanopeapods involve $\mathrm{C}_{60}$ fullerenes forming a chain inside a $\mathrm{BN}$ nanotube $[3,7,8]$, and these $\mathrm{BN}$ nanopeapods indicate potential applications as insulating nanowires. Furthermore, research on nanopeapods is also focused on the arrangement or packing of $\mathrm{C}_{60}$ fullerenes inside a carbon nanotube. Hodak and Girifalco $[9,10]$ find that the packing arrangement of $\mathrm{C}_{60}$ molecules inside a carbon nanotube strongly depends on the tube diameter and this is also the case for $\mathrm{BN}$ nanotubes [3]. However, the $\mathrm{C}_{60}-\mathrm{C}_{60}$ intermolecular distance inside a $\mathrm{BN}$ nanotube is $9 \AA$, which is slightly smaller than $10 \AA$, which is the corresponding distance inside a carbon nanotube $[3,11]$. Further, Okada et al [7] report that the encapsulation of $\mathrm{C}_{60}$ into a $(10,10)$ carbon nanotube is exothermic and that the energy gained in forming the peapod is $0.51 \mathrm{eV}$ per $\mathrm{C}_{60}$ molecule, but for $(8,8)$ and $(9,9)$ carbon nanotubes it is endothermic due to the large structural deformation of both tubes and fullerenes. For BN nanopeapods, Okada et al [12] find that the incorporations of $\mathrm{C}_{60}$ into both $(10,10)$ and $(9,9)$ BN nanotubes are exothermic, and that the energy gained upon formation of peapods is $1.3 \mathrm{eV}$ and $0.1 \mathrm{eV}$ respectively, which are both larger than that for a carbon nanopeapod. Also, Okada et al [12] mention that the structural deformations of both tubes and $\mathrm{C}_{60}$ fullerenes due to the encapsulation are smaller than those in carbon nanopeapods. Another issue regarding the formation of nanopeapods is the encapsulation of the $\mathrm{C}_{60}$ into the interior of a carbon nanotube. While Ulbricht and Hertel [13] and Ulbricht et al [14] suggest that a $\mathrm{C}_{60}$ is likely to be encapsulated head-on through the tube's open end, Berber et al [15] propose that a $\mathrm{C}_{60}$ molecule is most likely to be accepted into the tube via a large defect opening on the nanotube wall. However, Mickelson et al [3] suggest that $\mathrm{C}_{60}$ molecules mostly enter $\mathrm{BN}$ nanotubes through the ends of the tube, and not through the defects on the side walls.

In this paper, we consider the encapsulation of a $\mathrm{C}_{60}$ fullerene inside carbon and $\mathrm{BN}$ nanotubes as well as the encapsulation of a $\mathrm{B}_{36} \mathrm{~N}_{36}$ fullerene inside both carbon and $\mathrm{BN}$ nanotubes. We choose to study the $\mathrm{B}_{36} \mathrm{~N}_{36}$ fullerene, since it has an approximate diameter of 7-8 $\AA$ [4], which is similar to that of $\mathrm{C}_{60}$, and as pointed out by Hirano et al [4], Zope et al [16], Batsta et al [17], Alexandre et al [18] and Oku et al [19, 20] $\mathrm{B}_{36} \mathrm{~N}_{36}$ are the fullerenes that are usually observed in experiments to synthesize BN clusters, and once formed, the molecules are both energetically and vibrationally stable. For simplicity for the mathematical modelling, we assume a spherical shape for the $\mathrm{B}_{36} \mathrm{~N}_{36}$ fullerene.

The interaction between fullerenes and nanotubes has also led to the creation of gigahertz oscillators. From the experiments of Cumings and Zettl [21], Yu et al [22] and a number of molecular dynamics simulations [23-27], it is established that the sliding of an inner tube inside an outer tube of a multi-walled carbon nanotube can generate oscillatory frequencies in the gigahertz range. Further, Zheng et al [25] indicate that the shorter the inner tube, the higher the oscillatory frequency. This suggestion leads Liu et al [28] to the molecular dynamics study of a $\mathrm{C}_{60}$ oscillating inside a single-walled carbon nanotube. This latter study confirms that the oscillation of $\mathrm{C}_{60}$ inside a carbon nanotube results in a much higher frequency than that of the multi-walled carbon nanotube. 


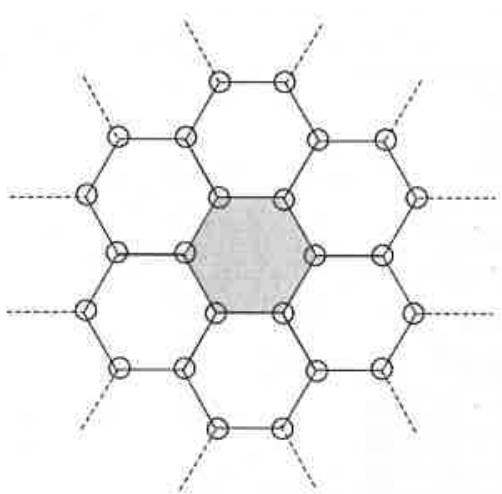

Figure 1. Hexagonal lattices on surface of nanotubes.

\section{Lennard-Jones potential}

To obtain the interaction energy between two non-bonded molecules, here we adopt the continuum approach which assumes a uniform distribution of atoms over the surface of each molecule. As such, instead of summing the potential interactions for each atom pair, the total interaction energy can be obtained by performing double surface integrals averaging over the surface of each entity, namely

$$
E=\eta_{1} \eta_{2} \int_{\Sigma_{1}} \int_{\Sigma_{2}} \Phi(r) \mathrm{d} \Sigma_{1} \mathrm{~d} \Sigma_{2}
$$

where $\eta_{1}$ and $\eta_{2}$ denote the mean surface density of atoms on each molecule and $r$ is the distance between two typical surface elements $d \Sigma_{1}$ and $d \Sigma_{2}$ on each molecule. We note that the average atomic density constants $\eta_{1}$ and $\eta_{2}$ are simply the number of atoms divided by the surface area of the molecule. For example, the mean atomic densities for $\mathrm{C}_{60}$ and $\mathrm{B}_{36} \mathrm{~N}_{36}$ spherical fullerenes are given respectively by $60 /\left(4 \pi b_{1}^{2}\right)$ and $72 /\left(4 \pi b_{2}^{2}\right)$, where $b_{1}$ and $b_{2}$ are radii of the $\mathrm{C}_{60}$ and $\mathrm{B}_{36} \mathrm{~N}_{36}$ fullerenes, respectively. For carbon and boron nitride nanotubes, we assume that their mean atomic densities are the same as those of their parent materials, namely graphite and h-BN, whose surfaces comprise hexagonal lattices as shown in figure 1 . We consider a unit hexagonal cell shown shaded in the figure with area $3 \sqrt{3} \sigma_{i}^{2} / 2$, where $\sigma_{1}$ and $\sigma_{2}$ denote the carbon-carbon and boron-nitrogen bond lengths (hexagonal side lengths), respectively. As shown, each atom participates in three cells, contributing one-third to each cell. Therefore, the mean atomic density is given by $(6 / 3) /\left(3 \sqrt{3} \sigma_{i}^{2} / 2\right)=4 \sqrt{3} /\left(9 \sigma_{i}^{2}\right)$ for carbon and boron nitride nanotubes. We comment that the continuum approximation represents an averaging procedure and might be expected to be mostly applicable to well defined molecular shapes, such as the cylindrical nanotubes and spherical fullerenes studied here. For non-regular shaped molecules, the continuum approach may not be an accurate approximation due to the difficulty in the determination of a mean atomic density. However, it is possible to combine both continuum and discrete approaches to model an interaction between regular and non-regular shaped nanostructures. As shown in both Hilder and Hill [29] and Verberck and Michel [30] for fullerene-nanotube interactions, the single-walled carbon nanotube is modelled as a continuum, while the fullerene is assumed to retain its discrete molecular structure.

The potential function adopted in this paper is the classical Lennard-Jones potential for two atoms at a distance $r$ apart, which is given by

$$
\Phi(r)=-A r^{-6}+B r^{-12}
$$

where $A$ and $B$ are the attractive and repulsive constants, respectively. Equation (2.2) can also 
Table 1. Values of constants used in this paper.

\begin{tabular}{|c|c|}
\hline Radius of $\mathrm{C}_{60}$ & $b_{1}=3.55 \AA$ \\
\hline Average radius of $\mathrm{B}_{36} \mathrm{~N}_{36}$ & $b_{2}=3.50 \AA$ \\
\hline $\mathrm{C}-\mathrm{C}$ bond length & $\sigma_{1}=1.421 \AA$ \\
\hline $\mathrm{B}-\mathrm{N}$ bond length & $\sigma_{2}=1.446 \AA$ \\
\hline Mean surface density for $\mathrm{C}_{60}\left[60 /\left(4 \pi b_{1}^{2}\right)\right]$ & $\eta_{f 1}=0.3789 \AA^{-2}$ \\
\hline Mean surface density for $\mathrm{B}_{36} \mathrm{~B}_{36}\left[72 /\left(4 \pi b_{2}^{2}\right)\right]$ & $\eta_{f 2}=0.4677 \AA^{-2}$ \\
\hline Mean surface density for CNT $\left[4 \sqrt{3} /\left(9 \sigma_{1}^{2}\right)\right]$ & $\eta_{11}=0.3812 \AA^{-2}$ \\
\hline Mean surface density for BNNT $\left[4 \sqrt{3} /\left(9 \sigma_{2}^{2}\right)\right]$ & $\eta_{12}=0.3682 \AA^{-2}$ \\
\hline Mass of a single $C$ atom & $m_{1}=19.92 \times 10^{-27} \mathrm{~kg}$ \\
\hline Mass of a single $B$ atom & $m_{2}=17.95 \times 10^{-27} \mathrm{~kg}$ \\
\hline Mass of a single $\mathrm{N}$ atom & $m_{3}=23.25 \times 10^{-27} \mathrm{~kg}$ \\
\hline Mass of a single $\mathrm{C}_{60}\left[60 \mathrm{~m}_{1}\right]$ & $M_{1}=1195.2 \times 10^{-27} \mathrm{~kg}$ \\
\hline Mass of a single $\mathrm{B}_{36} \mathrm{~N}_{36}\left[36\left(m_{2}+m_{3}\right)\right]$ & $M_{2}=1483.1 \times 10^{-27} \mathrm{~kg}$ \\
\hline Attractive constant for $\mathrm{C}-\mathrm{C}$ interaction & $A_{\mathrm{C}}=15.41 \mathrm{eV} \AA^{6}$ \\
\hline Repulsive constant for $\mathrm{C}-\mathrm{C}$ interaction & $B_{C}=22534.75 \mathrm{eV} \AA^{12}$ \\
\hline Attractive constant for B-B interaction & $A_{B}=27.91 \mathrm{eVA}^{6}$ \\
\hline Repulsive constant for B-B interaction & $B_{\mathrm{B}}=47303.61 \mathrm{eV} \AA^{12}$ \\
\hline Attractive constant for $\mathrm{N}-\mathrm{N}$ interaction & $A_{\mathrm{N}}=36.48 \mathrm{eV} \AA^{6}$ \\
\hline Repulsive constant for $\mathrm{N}-\mathrm{N}$ interaction & $B_{\mathrm{N}}=52955.32 \mathrm{eV \AA ^{12 }}$ \\
\hline Attractive constant for $\mathrm{C}-\mathrm{B}$ interaction & 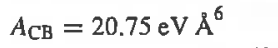 \\
\hline Repulsive constant for $\mathrm{C}-\mathrm{B}$ interaction & $B_{\mathrm{CB}}=32678.99 \mathrm{eV} \AA^{12}$ \\
\hline Attractive constant for $\mathrm{C}-\mathrm{N}$ interaction & $A_{\mathrm{CN}}=23.71 \mathrm{eV} \AA^{6}$ \\
\hline Repulsive constant for $\mathrm{C}-\mathrm{N}$ interaction & $B_{\mathrm{CN}}=34544.75 \mathrm{eV} \AA^{12}$ \\
\hline Attractive constant for $\mathrm{B}-\mathrm{N}$ interaction & $A_{\mathrm{BN}}=31.92 \mathrm{eV}^{6}$ \\
\hline Repulsive constant for $\mathrm{B}-\mathrm{N}$ interaction & $B_{\mathrm{BN}}=50099.81 \mathrm{eV}^{12}$ \\
\hline
\end{tabular}

be written in terms of the energy well depth $\epsilon$ and the van der Waals diameter $\sigma$ given by

$$
\Phi(\rho)=4 \epsilon\left[-\left(\frac{\sigma}{r}\right)^{6}+\left(\frac{\sigma}{r}\right)^{12}\right],
$$

noting that $\epsilon=A^{2} /(4 B)$ and the equilibrium distance $r_{0}$ between the two atoms is given by $r_{0}=2^{1 / 6} \sigma=(2 B / A)^{1 / 6}$. Following Lee [31] and Kang and Hwang [32], the LennardJones parameters used in this paper are given by $\epsilon_{\text {carbon }}=0.002635 \mathrm{eV}, \sigma_{\text {carbon }}=3.369 \AA$, $\epsilon_{\text {boron }}=0.004116 \mathrm{eV}, \sigma_{\text {boron }}=3.453 \AA, \epsilon_{\text {nitrogen }}=0.006281 \mathrm{eV}$ and $\sigma_{\text {nirogen }}=3.365 \AA$. Further, the non-bonded carbon-boron, carbon-nitrogen and boron-nitrogen parameters are obtained using the Lorentz-Berthelot mixing rules, $\epsilon_{a b}=\sqrt{\epsilon_{a} \epsilon_{b}}$ and $\sigma_{a b}=\left(\sigma_{a}+\sigma_{b}\right) / 2$. From these parameter values, we are able to derive the attractive and repulsive Lennard-Jones constants $A$ and $B$ for each molecular interaction as given in table 1 .

In Cox et al [33,34], the interaction force and energy between a $\mathrm{C}_{60}$ fullerene and a carbon nanotube is investigated using (2.1) and (2.2). While Cox et al [33] focus on the interaction between a $\mathrm{C}_{60}$ molecule and a carbon nanotube, in general their mathematical expressions can be applied for any spherical cage-like molecule (fullerene) interacting with a cylindrical nanotube of any kind. Note that the parameter values, including radii of nanotube and fullerene $a$ and $b$, the attractive and repulsive constants $A$ and $B$ and the mean atomic surface densities of the fullerene and the nanotube, $\eta_{f}$ and $\eta_{t}$, change according to the interacting molecules under consideration. In table 1, we summarize the numerical values of the constants which are used throughout this paper. 
In this paper, we adopt the Girifalco [35] and Cox et al [33, 34] continuum models and extend the study for the interactions of a boron nitride nanotube and a boron nitride $\mathrm{B}_{36} \mathrm{~N}_{36}$ fullerene. In fact, we consider the following interactions: $\mathrm{C}_{60}-\mathrm{C}_{60}, \mathrm{~B}_{36} \mathrm{~N}_{36}-\mathrm{B}_{36} \mathrm{~N}_{36}$, $\mathrm{C}_{60}-\mathrm{B}_{36} \mathrm{~N}_{36}, \mathrm{C}_{60}$-carbon nanotube, $\mathrm{C}_{60}$-boron nitride nanotube, $\mathrm{B}_{36} \mathrm{~N}_{36}$-carbon nanotube and $\mathrm{B}_{36} \mathrm{~N}_{36}$-boron nitride nanotube. The results obtained from the continuum based model are then compared with molecular dynamics studies previously presented in the literature. We note here that while most calculations in the area of molecular interactions are done through large scale computations, our contribution is to provide an understanding of the complex problems through the use of elementary physics and classical mathematical modelling.

\section{Interacting fullerenes}

Here, we examine the interactions between two fullerenes. Girifalco [35] gives an energy function for two identical spherical fullerenes based on the Lennard-Jones potential and the continuum approximation. Following Ruoff and Hickman [36] and Girifalco [35], the interaction potential energy $E$ between two non-identical and non-concentric spherical fullerenes can be obtained as

$$
\begin{aligned}
& E=\eta_{1} \eta_{2}\left[-A P_{6}+B P_{12}\right], \\
& P_{n}=\frac{4 \pi^{2} b c}{\rho(2-n)(3-n)}\left\{\frac{1}{(b+c+\rho)^{n-3}}+\frac{1}{(b-c-\rho)^{n-3}}\right. \\
& \left.\quad-\frac{1}{(b+c-\rho)^{n-3}}-\frac{1}{(b-c+\rho)^{n-3}}\right\},
\end{aligned}
$$

where $b$ and $c$ are radii of the two interacting fullerenes, $\rho$ here denotes the distance between their centres, $\eta_{1}$ and $\eta_{2}$ are the mean atomic densities of the fullerenes and $A$ and $B$ are the Lennard-Jones attractive and repulsive constants, respectively.

We note that the above equations have also been previously derived by Iglesias-Groth et al [37]. For $\mathrm{C}_{60}-\mathrm{C}_{60}$ interaction, we simply obtain the energy $E$ by using (3.1), (3.2) and the numerical values of the constants shown in table 1 , where $A=A_{C}, B=B_{\mathrm{C}}$ and $\eta_{1}=\eta_{2}=\eta_{f 1}$, which is the mean surface density of the fullerene $\mathrm{C}_{60}$. However, for the case of $\mathrm{C}_{60}-\mathrm{B}_{36} \mathrm{~N}_{36}$ we assume that the $\mathrm{C}_{60}$ interacts with one half of the molecule which has 36 boron atoms and another half of the molecule which has 36 nitrogen atoms, so that the energy can be obtained as $E=\eta_{f 1}\left(\eta_{f 2} / 2\right)\left[-A_{\mathrm{CB}} P_{6}+B_{\mathrm{CB}} P_{12}\right]+\eta_{f 1}\left(\eta_{f 2} / 2\right)\left[-A_{\mathrm{CN}} P_{6}+B_{\mathrm{CN}} P_{12}\right]$, where $\eta_{f 2}$ denotes the mean atomic surface density of the fullerene $B_{36} \mathrm{~N}_{36}$ and $\eta_{1}=\eta_{f 1}$. Similarly for $\mathrm{B}_{36} \mathrm{~N}_{36}-\mathrm{B}_{36} \mathrm{~N}_{36}$ we have four interaction components, namely $\mathrm{B}-\mathrm{B}, \mathrm{B}-\mathrm{N}, \mathrm{N}-$ $\mathrm{B}$ and N-N, thus $E=\left(\eta_{f 1} / 2\right)\left(\eta_{f 2} / 2\right)\left[-A_{\mathrm{B}} P_{6}+B_{\mathrm{B}} P_{12}\right]+2\left(\eta_{f 1} / 2\right)\left(\eta_{f 2} / 2\right)\left[-A_{\mathrm{BN}} P_{6}+\right.$ $\left.B_{\mathrm{BN}} P_{12}\right]+\left(\eta_{f 1} / 2\right)\left(\eta_{f 2} / 2\right)\left[-A_{\mathrm{N}} P_{6}+B_{\mathrm{N}} P_{12}\right]$, where $\eta_{f}=\eta_{f 1}=\eta_{f 2}$ is the mean surface density of $B_{36} N_{36}$. By using (3.1) and (3.2) and the constants given in table 1, we plot the interaction energies of $\mathrm{C}_{60}-\mathrm{C}_{60}, \mathrm{C}_{60}-\mathrm{B}_{36} \mathrm{~N}_{36}$ and $\mathrm{B}_{36} \mathrm{~N}_{36}-\mathrm{B}_{36} \mathrm{~N}_{36}$ with respect to the distance $\rho$, as shown in figure 2. From this figure and equations (3.1) and (3.2) we find that the equilibrium distances for $\mathrm{C}_{60}-\mathrm{C}_{60}, \mathrm{C}_{60}-\mathrm{B}_{36} \mathrm{~N}_{36}$ and $\mathrm{B}_{36} \mathrm{~N}_{36}-\mathrm{B}_{36} \mathrm{~N}_{36}$ interactions are 9.971, 9.937 and $9.903 \AA$, respectively. We note that the equilibrium distances are sensitive to the values of the Lennard-Jones constants used in the model. For example, if we use $A_{\mathrm{C}}=20.0 \mathrm{eV} \AA^{6}$ and $B_{\mathrm{C}}=34.8 \times 10^{3} \mathrm{eV} \AA^{12}$ as given by Girifalco et al [38] for the interaction between $\mathrm{C}_{60}$ and $\mathrm{C}_{60}$, then we may obtain $\rho_{0}=10.034 \AA$ as the equilibrium distance. Further, we also find from figure 2 that at equilibrium the $B_{36} \mathrm{~N}_{36}-\mathrm{B}_{36} \mathrm{~N}_{36}$ interaction has the lowest minimum energy, which therefore gives rise to the strongest interaction. 


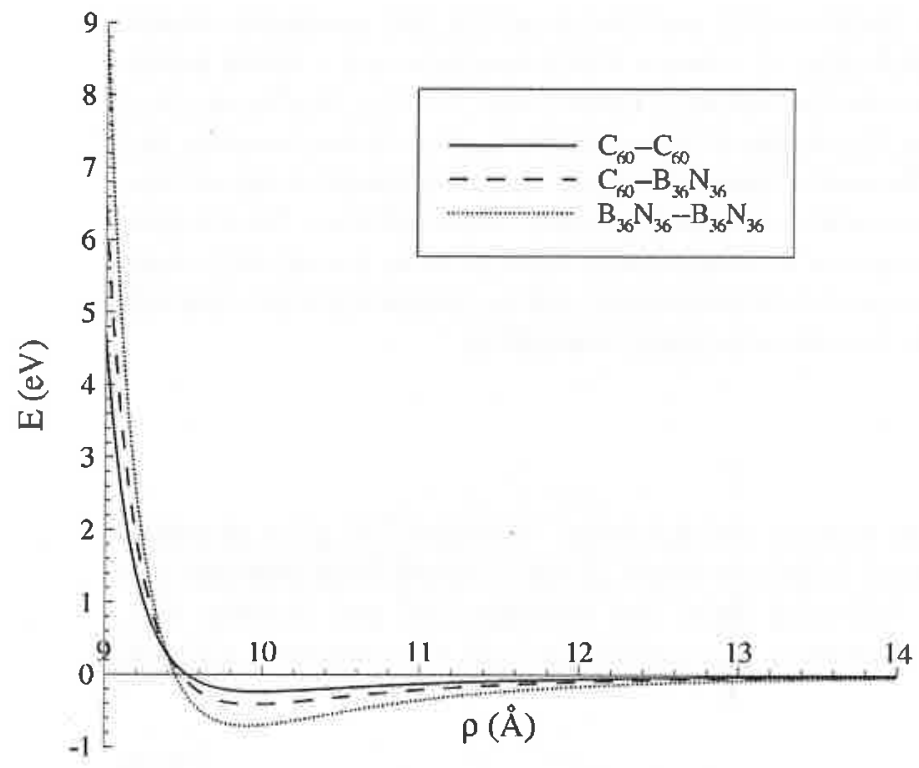

Figure 2. Interaction energy between two fullerenes at a distance $\rho$ apart.

\section{Interacting fullerenes and nanotubes}

In this section, we consider the interactions of fullerenes and nanotubes. The mechanics of the encapsulation of $\mathrm{C}_{60}$ into nanotubes is investigated using a molecular dynamics simulation [39]. For a $\mathrm{C}_{60}$ initially at rest in the vicinity of a carbon nanotube open end, this study suggests that $(9,9)$ and $(10,10)$ nanotubes will accept the molecule, whereas an $(8,8)$ nanotube will not. As shown in the simulations of Qian et al [39], the suction force of the $\mathrm{C}_{60}$ molecule by the carbon nanotube results in the oscillation of the molecule between the tube open ends. These findings lead $\mathrm{Cox}$ et al $[33,34]$ to study the problem of suction of a $\mathrm{C}_{60}$ fullerene and the molecular oscillation inside a single-walled carbon nanotube. While experimental and highly computational studies are usually employed in this area, Cox et al $[33,34]$ adapt mechanical principles and mathematical modelling techniques to provide a model which provides considerable insight into the problem. In particular, Cox et al $[33,34]$ adopt the Lennard-Jones potential together with the continuum approach to obtain the interaction force and energy between a $\mathrm{C}_{60}$ molecule and a carbon nanotube. Their results indicate that a nanotube with radius smaller than $6.338 \AA$ will not accept the $\mathrm{C}_{60}$ from rest. This result is in excellent agreement with Okada et al [7] and Hodak and Girifalco [40], who predict 6.4 and $6.27 \AA$ respectively for the minimum radius of a carbon nanotube that will accept the $\mathrm{C}_{60}$ molecule, whereas it contradicts Qian et al $[39]$ that a $(9,9)$ nanotube of radius $6.106 \AA$ will accept the $\mathrm{C}_{60}$ from rest.

In this paper, we further investigate the issues of acceptance and suction energies for the cases $\mathrm{C}_{60}$-boron nitride nanotube, $\mathrm{B}_{36} \mathrm{~N}_{36}$-carbon nanotube and $\mathrm{B}_{36} \mathrm{~N}_{36}$-boron nitride nanotube. From Cox et al [33], with the use of (2.1) and (2.2), it is shown that the total axial force for a fullerene interacting with a nanotube, as shown in figure 3 , is given by

$F^{*}=\frac{8 \pi^{2} \eta_{1} \eta_{2} a}{b^{4} \lambda^{3}}\left[A\left(1+\frac{2}{\lambda}\right)-\frac{B}{5 b^{6} \lambda^{3}}\left(5+\frac{80}{\lambda}+\frac{336}{\lambda^{2}}+\frac{512}{\lambda^{3}}+\frac{256}{\lambda^{4}}\right)\right]$,

where $\lambda=\left(a^{2}-b^{2}+Z^{2}\right) / b^{2}$. Similar to the case of two interacting fullerenes, the total axial forces for $\mathrm{C}_{60}-\mathrm{BN}$ nanotube, $\mathrm{B}_{36} \mathrm{~N}_{36}$-carbon nanotube and $\mathrm{B}_{36} \mathrm{~N}_{36}-\mathrm{BN}$ nanotube can be given 




Figure 3. Fullerene entering the open end of a nanotube.

Table 2. Critical values $a_{\mathrm{c}}$ for each interaction.

\begin{tabular}{lllll}
\hline & \multicolumn{5}{c}{ Interactions } \\
\cline { 2 - 5 } & $\mathrm{C}_{60}-\mathrm{CNT}$ & $\mathrm{C}_{60}-\mathrm{BNNT}$ & $\mathrm{B}_{36} \mathrm{~N}_{36}-\mathrm{CNT}$ & $\mathrm{B}_{36} \mathrm{~N}_{36}-\mathrm{BNNT}$ \\
\hline$a_{\mathrm{c}}(\mathrm{A})$ & 6.445 & 6.461 & 6.411 & 6.427 \\
\hline
\end{tabular}

respectively as

$$
\begin{aligned}
F=F^{*}\left(\eta_{1}=\right. & \left.\eta_{f 1}, \eta_{2}=\eta_{t 2} / 2, A=A_{\mathrm{CB}}, B=B_{\mathrm{CB}}\right) \\
& +F^{*}\left(\eta_{1}=\eta_{f 1}, \eta_{2}=\eta_{t 2} / 2, A=A_{\mathrm{CN}}, B=B_{\mathrm{CN}}\right), \\
F=F^{*}\left(\eta_{1}=\right. & \left.\eta_{f 2} / 2, \eta_{2}=\eta_{t 1}, A=A_{\mathrm{CB}}, B=B_{\mathrm{CB}}\right) \\
& +F^{*}\left(\eta_{1}=\eta_{f 2} / 2, \eta_{2}=\eta_{t 1}, A=A_{\mathrm{CN}}, B=B_{\mathrm{CN}}\right), \\
F=F^{*}\left(\eta_{1}=\right. & \left.\eta_{f 2} / 2, \eta_{2}=\eta_{t 2} / 2, A=A_{\mathrm{B}}, B=B_{\mathrm{B}}\right) \\
& +F^{*}\left(\eta_{1}=\eta_{f 2} / 2, \eta_{2}=\eta_{t 2} / 2, A=A_{\mathrm{N}}, B=B_{\mathrm{N}}\right) \\
& +2 F^{*}\left(\eta_{1}=\eta_{f 2} / 2, \eta_{2}=\eta_{t 2} / 2, A=A_{\mathrm{BN}}, B=B_{\mathrm{BN}}\right),
\end{aligned}
$$

where these constants are as given in table 1 .

Upon plotting $F$ with respect to the position $Z$ of the fullerene as shown in figure 4, it can be seen that the total axial force for each interaction of $\mathrm{C}_{60}-\mathrm{BN}$ nanotube, $\mathrm{B}_{36} \mathrm{~N}_{36}-$ carbon nanotube and $\mathrm{B}_{36} \mathrm{~N}_{36}-\mathrm{BN}$ nanotube behaves in a similar manner. This behaviour is also similar to that shown in Cox et al [33] for $\mathrm{C}_{60}$-carbon nanotube, which is also presented here in figure 4(a). From figure 4, $F$ is continuous and strongly dependent on the nanotube radius $a$. As the radius of the tube increases beyond a critical value $a_{\mathrm{c}}$, the value of $F$ remains positive for all values of $Z$. This implies that a nanotube with $a \geqslant a_{\mathrm{c}}$ will accept the fullerene from rest. In table 2, we present the values $a_{\mathrm{c}}$ for each of the interacting configurations.

Next, we determine the minimum radius $\left(a_{\mathrm{m}}<a_{\mathrm{c}}\right)$ of a nanotube that will still accept a fullerene from rest. As seen in figure 4, when the value of $a$ is less than the critical value $a_{\mathrm{c}}$ equation $F(Z)=0$ has at most two real roots $Z= \pm Z_{0}$. If the integral of $F(Z)$ represents the work imparted to the fullerene and can be equated directly to the kinetic energy, then the acceptance energy can be defined as $W_{a}=\int_{-\infty}^{Z_{0}} F(Z) \mathrm{d} Z$. From the $\mathrm{C}_{60}$-carbon nanotube interaction as shown in Cox et al [33], we have

$W_{a}=\frac{8 \pi^{2} \eta_{f} \eta_{t} a}{b^{2} \sqrt{a^{2}-b^{2}}}\left[A\left(I_{2}+2 I_{3}\right)-\frac{B}{5 b^{6}}\left(5 I_{5}+80 I_{6}+336 I_{7}+512 I_{8}+256 I_{9}\right)\right]$,

where $I_{n}=b^{2 n}\left(a^{2}-b^{2}\right)^{-n} \int_{-\pi / 2}^{\psi_{0}} \cos ^{2 n} \psi \mathrm{d} \psi$ and $\psi_{0}=\tan ^{-1}\left(Z_{0} / \sqrt{a^{2}-b^{2}}\right)$. As such, with the use of (4.5) and similar approaches to that of (4.2), (4.3) and (4.4), we can determine $W_{a}$ for the $\mathrm{C}_{60}-\mathrm{BN}$ nanotube, the $\mathrm{B}_{36} \mathrm{~N}_{36}$-carbon nanotube and the $\mathrm{B}_{36} \mathrm{~N}_{36}-\mathrm{BN}$ nanotube interactions respectively. The acceptance energy must be positive for a nanotube to accept a fullerene 


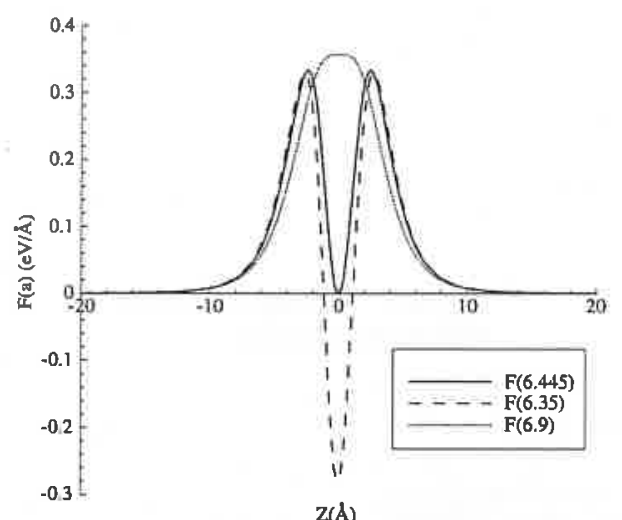

(a).

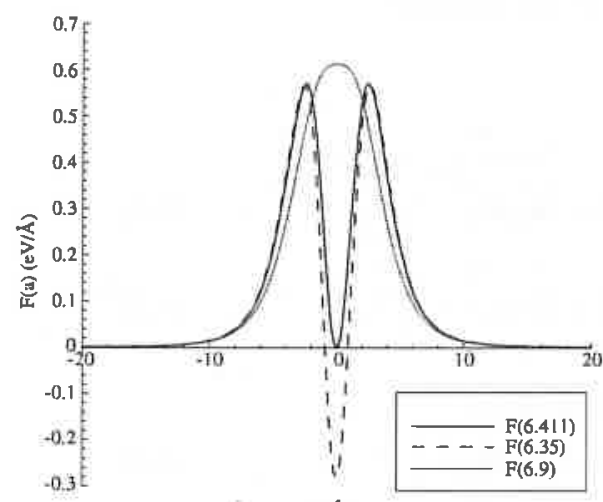

(c).

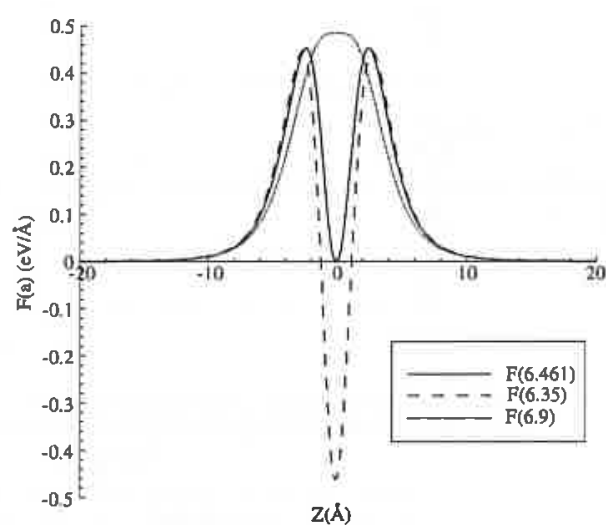

(b).

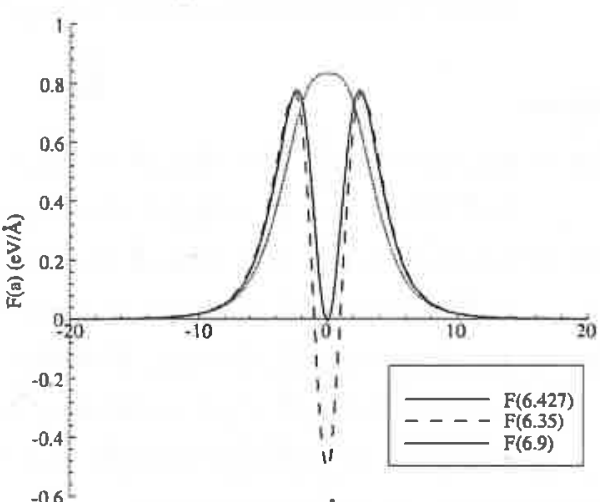

(d).

Figure 4. Force experienced by a fullerene due to van der Waals interaction with a semi-infinite nanotube: (a) $\mathrm{C}_{60}$-carbon nanotube, (b) $\mathrm{C}_{60}-\mathrm{BN}$ nanotube, (c) $\mathrm{B}_{36} \mathrm{~N}_{36}$-carbon nanotube and (d) $\mathrm{B}_{36} \mathrm{~N}_{36}-\mathrm{BN}$ nanotube.

Table 3. Minimum radius $a_{\mathrm{m}}$ of a nanotube that will accept a fullerene from rest.

\begin{tabular}{lllll}
\hline & \multicolumn{5}{c}{ Interactions } \\
\cline { 2 - 5 } & $\mathrm{C}_{60}-\mathrm{CNT}$ & $\mathrm{C}_{60}-\mathrm{BNNT}$ & $\mathrm{B}_{36} \mathrm{~N}_{36}-\mathrm{CNT}$ & $\mathrm{B}_{36} \mathrm{~N}_{36}-\mathrm{BNNT}$ \\
\hline$a_{\mathrm{m}}(\AA)$ & 6.277 & 6.293 & 6.243 & 6.258 \\
\hline
\end{tabular}

initially at rest at the tube open end. If $W_{a}$ is negative, then its magnitude represents the initial kinetic energy needed by the fullerene in the form of the inbound initial velocity for it to be to accepted into the nanotube. In figure 5, we plot $W_{a}$ with respect to the radius of a nanotube for each interaction and in table 3 we present the values of the radii of a nanotube, $a_{\mathrm{m}}$, for which $W_{a}=0$. We note that nanotubes with radii smaller than $a_{m}$ will not accept fullerenes which are initially at rest by suction forces alone. Although the same model is used but with different values of the contants $A_{\mathrm{C}}$ and $B_{\mathrm{C}}$, the results here and that of Cox et al [33] for the $\mathrm{C}_{60}$-carbon nanotube are quite distinct. While the minimum radius of a carbon nanotube that will accept 


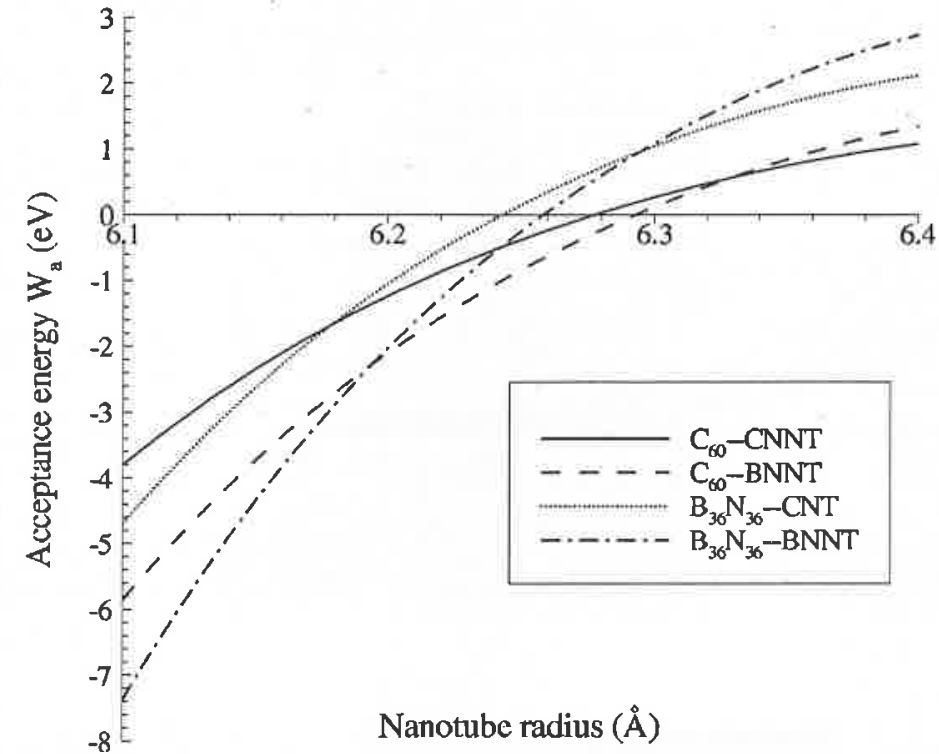

Figure 5. Acceptance energy threshold for a fullerene to be sucked into a nanoube.

Table 4. Optimal radius of nanotubes $a_{\max }$ which lead to the maximal suction energies $W_{\max }$.

\begin{tabular}{lllll}
\hline & \multicolumn{4}{c}{ Interactions } \\
\cline { 2 - 5 } & $\mathrm{C}_{60}-\mathrm{CNT}$ & $\mathrm{C}_{60}-\mathrm{BNNT}$ & $\mathrm{B}_{36} \mathrm{~N}_{36}-\mathrm{CNT}$ & $\mathrm{B}_{36} \mathrm{~N}_{36}-\mathrm{BNNT}$ \\
\hline$a_{\max }(\AA)$ & 6.713 & 6.730 & 6.681 & 6.699 \\
$W_{\max }(\mathrm{eV})$ & 3.089 & 4.223 & 5.297 & 7.241 \\
\hline
\end{tabular}

a fullerene $C_{60}$ determined here is $6.277 \AA$, Cox et al [33] determine $6.338 \AA$. However, both results agree well with the 6.27 and $6.4 \AA$ predicted by Hodak and Girifalco [40] and Okada et al [7], respectively. Comparing the acceptance of the same molecule, table 3 shows that the required minimum size is smaller for a carbon nanotube than a $\mathrm{BN}$ nanotube.

Following [33], $W$ denotes the suction energy for a fullerene, which is the total work performed by the van der Waals interactions on a fullerene entering a carbon nanotube. The suction energy can be represented mathematically as $W=\int_{-\infty}^{\infty} F(Z) \mathrm{d} Z$, and as derived by Cox et al [33] it is given by

$W=\frac{\pi^{3} \eta_{f} \eta_{t} a b^{2}}{\left(a^{2}-b^{2}\right)^{5 / 2}}\left[A(3+5 \mu)-\frac{B\left(315+4620 \mu+18018 \mu^{2}+25740 \mu^{3}+12155 \mu^{4}\right)}{160\left(a^{2}-b^{2}\right)^{3}}\right]$,

where $\mu=b^{2} /\left(a^{2}-b^{2}\right)$. Again, using (4.6) and the approaches as of (4.2), (4.3) and (4.4), we obtain the $\mathrm{C}_{60}-\mathrm{BN}$ nanotube, $\mathrm{B}_{36} \mathrm{~N}_{36}$-carbon nanotube and $\mathrm{B}_{36} \mathrm{~N}_{36}-\mathrm{BN}$ nanotube, respectively. In figure 6, we illustrate graphically the relation between the suction energy and the nanotube radius for each interaction type. This figure shows for each interaction that there is an optimal radius $a_{\max }$ for a nanotube that maximizes the suction energy. In table 4 , the values of $a_{\max }$ that give rise to $W_{\max }$ for each interaction are presented. We comment that since the suction energy can be equated directly to the kinetic energy of the molecule, for the fullerene to travel at the maximum velocity, the optimal size nanotube which is indicated in table 4 must be adopted. From this table, we find that the greatest velocity is obtained from a $\mathrm{B}_{36} \mathrm{~N}_{36}$ 


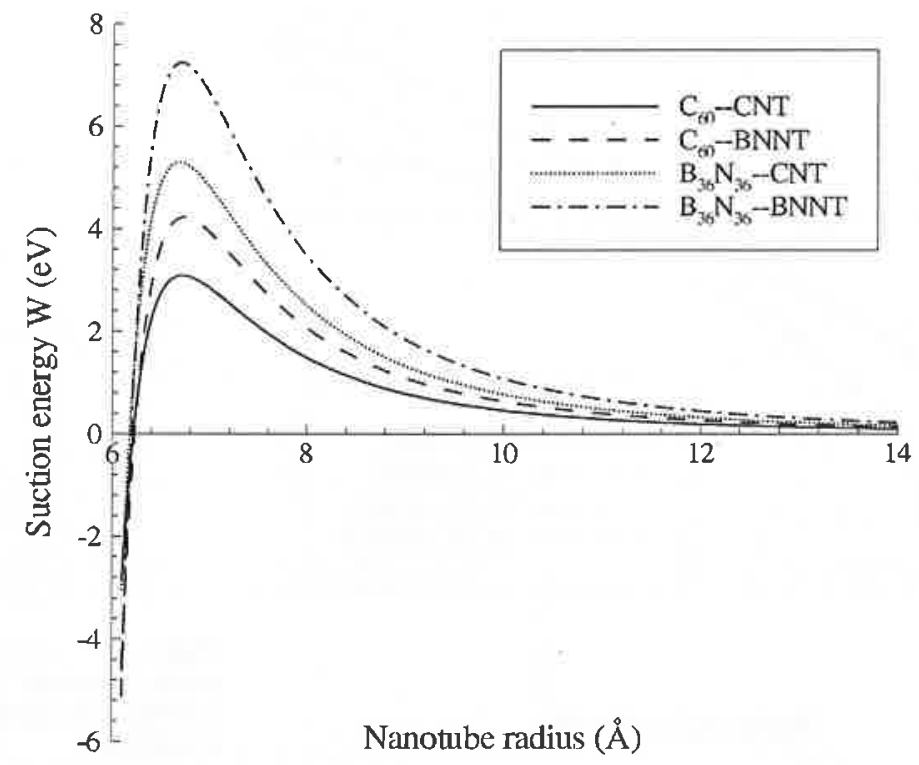

Figure 6. Suction energy for a fullerene entering a nanotube.

travelling inside a $\mathrm{BN}$ nanotube. As shown, we predict the formation energy (referred to here as the suction energy) of a $\mathrm{C}_{60} @ \mathrm{CNT}$ as $3.089 \mathrm{eV}$, which is in close agreement with molecular dynamics studies of Ulbricht et al [14] and Kang and Hwang [32], who predict 3.01 and $3.02 \mathrm{eV}$, respectively. Our result also shows a reasonable agreement with Girifalco et al [38], who predict $3.26 \mathrm{eV}$ for the formation energy of a $\mathrm{C}_{60} @ \mathrm{CNT}$. Further, our prediction of $4.223 \mathrm{eV}$ for the formation energy of $\mathrm{C}_{60} @ \mathrm{BNNT}$ is again in excellent agreement with Kang and Hwang [32], who predict $4.383 \mathrm{eV}$. We comment that, although we cannot find any experimental or computational results for $\mathrm{B}_{36} \mathrm{~N}_{36} @ \mathrm{CNT}$ and $\mathrm{B}_{36} \mathrm{~N}_{36} @ \mathrm{BNNT}$, the above comparisons validate our model.

\section{Offset location of fullerenes inside nanotubes}

Inside a nanotube, a molecule tends be at an inter-atomic distance away from the nanotube wall. This preferred position is where the interaction energy between the molecule and the nanotube is a minimum. In Cox et al [34], it is predicted that a $\mathrm{C}_{60}$ molecule is likely to be on the central tube axis of a $(10,10)$ nanotube, and as a carbon nanotube becomes larger, the preferred position tends to be offset further away from the tube axis. The result of Cox et al [34] is consistent with Hodak and Girifalco $[9,10]$ and others that $C_{60}$ molecules inside a $(10,10)$ nanotube tend to form a linear chain along the tube axis, whereas in $(15,15)$ the $\mathrm{C}_{60}$ molecules are offset from the axis and form a zigzag chain. In this paper, we adopt the interaction potential function for an offset $\mathrm{C}_{60}$ fullerene and a single-walled carbon nanotube derived in Cox et al [34] to determine the preferred position of an inner molecule inside a nanotube. The inner molecules studied here comprise $\mathrm{C}_{60}$ and $\mathrm{B}_{36} \mathrm{~N}_{36}$ fullerenes and the nanotubes considered are carbon and boron nitride.

If we assume that the fullerene is offset away from the nanotube central axis by $\varepsilon$ as shown in figure 7, then from Cox et al [34] the potential for the offset $\mathrm{C}_{60}$ molecule inside the singlewalled carbon nanotube of infinite length is given by 


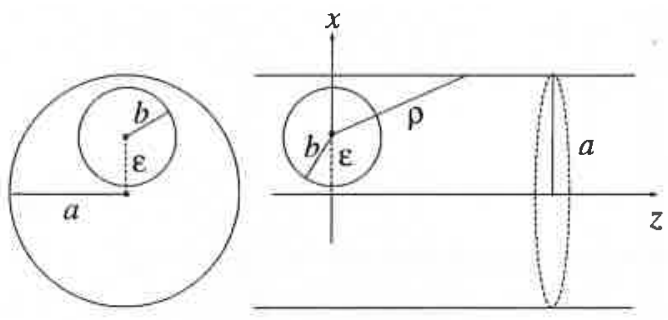

Figure 7. An offset fullerene inside a nanotube.

$$
\begin{aligned}
E=4 \pi^{2} a b^{2} \eta_{f} \eta_{t} & {\left[\frac{B}{5}\left(\frac{315}{256} J_{5}+\frac{1155 b^{2}}{64} J_{6}+\frac{9009 b^{4}}{128} J_{7}+\frac{6435 b^{6}}{64} J_{8}+\frac{12155 b^{8}}{256} J_{9}\right)\right.} \\
- & \left.\frac{A}{8}\left(3 J_{2}+5 b^{2} J_{3}\right)\right],
\end{aligned}
$$

where the integrals $J_{n}$ are defined by

$$
J_{n}=\int_{-\pi}^{\pi} \frac{\mathrm{d} \theta}{(\alpha-\beta \cos \theta)^{n+1 / 2}},
$$

and $\alpha=a^{2}+\varepsilon^{2}-b^{2}$ and $\beta=2 a \varepsilon$. We note that (5.1) corrects a minor typographical error reported in [34], where the factor $315 / 256$ is incorrectly stated as $105 / 128$. As shown in Cox et al [34], this integral can be evaluated in terms of the hypergeometric function $F(p, q ; r ; z)$ as

$$
J_{n}=\frac{2 \pi}{\gamma^{m}} F(m, 1 / 2 ; 1 ;-\omega / \gamma),
$$

where $m=n+1 / 2, \gamma=\alpha-\beta$ and $\omega=2 \beta$. The constants $a$ and $b$ are the radii of the nanotube and the fullerene, respectively, and $A$ and $B$ denote the attractive and repulsive constants, respectively. We note that these are material parameters, and as mentioned previously (5.1) can be adopted for any spherical fullerene interacting with any nanotube, provided that these constants are known. Again, for the determination of the potential energy $E$ of an offset fullerene inside a nanotube we may adopt the same approach as shown in (4.2), (4.3) and (4.4) for $\mathrm{C}_{60}-\mathrm{BN}$ nanotube, $\mathrm{B}_{36} \mathrm{~N}_{36}$-carbon nanotube and $\mathrm{B}_{36} \mathrm{~N}_{36}-\mathrm{BN}$ nanotube, respectively.

Figure 8 shows the potential energy $E$ with respect to the radial distance $\varepsilon$ for the four types of interactions, namely $\mathrm{C}_{60}$-carbon nanotube, $\mathrm{C}_{60}$-boron nitride nanotube, $\mathrm{B}_{36} \mathrm{~N}_{36}-$ carbon nanotube and $\mathrm{B}_{36} \mathrm{~N}_{36}$-boron nitride nanotube. For each interaction, we determine the offset distance $\varepsilon$ for the inner molecules inside $(10,10),(15,15)$ and $(20,20)$ carbon and boron nitride nanotubes. We note that the radii of the nanotubes are determined from $a=a^{*} \sqrt{n^{2}+m^{2}+n m} /(2 \pi)$, where $(n, m)$ is the chiral vector and $a^{*}=2.456$ and $2.504 \AA$ for carbon and boron nitride nanotubes, respectively. Similarly to the $\mathrm{C}_{60}-$ carbon nanotube interaction shown in [34], the preferred position of a fullerene tends to be further away from the tube axis and closer to the tube wall as the nanotube radius increases. However, there is a particular size of nanotube where $\varepsilon=0$ and the centre of the fullerene is likely to lie on the $z$-axis. For all cases this particular size is equivalent to the size of a $(10,10)$ nanotube. From figure 7 , we note that the shortest distance between the surfaces of the fullerene and the nanotube is given by $d=a-(\varepsilon+b)$. The distances $d$ for $\mathrm{C}_{60}-(10,10) \mathrm{CNT}, \mathrm{C}_{60}-(10,10)$ BNNT, $\mathrm{B}_{36} \mathrm{~N}_{36}-(10,10) \mathrm{CNT}$ and $\mathrm{B}_{36} \mathrm{~N}_{36}-(10,10)$ BNNT are found to be $3.22,3.35,3.27$ and $3.40 \AA$, respectively. As expected, these distances are close to the interlayer distances of their parent materials, which are 3.35 for graphite and 3.33 for $\mathrm{h}-\mathrm{BN}$ [2]. We further comment that, as the van der Waals force dominates only at short ranges, inside a small tube (that is large enough to incorporate a fullerene), such as a $(10,10)$ nanotube, the fullerene interacts with the 


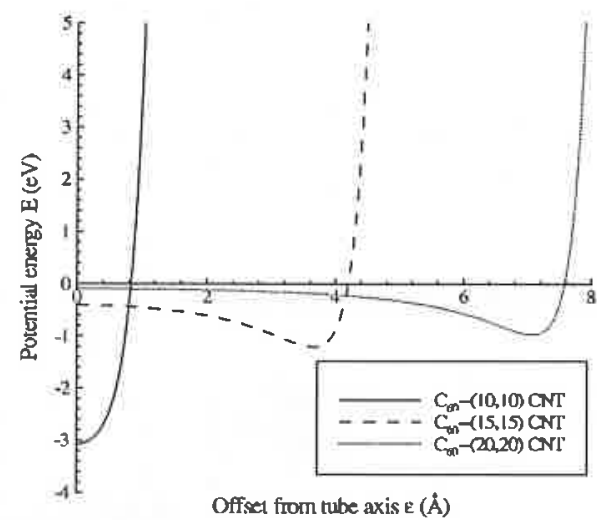

(a).

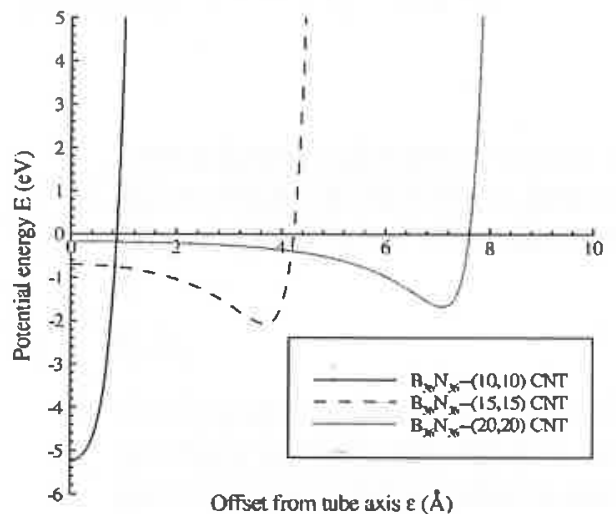

(c).

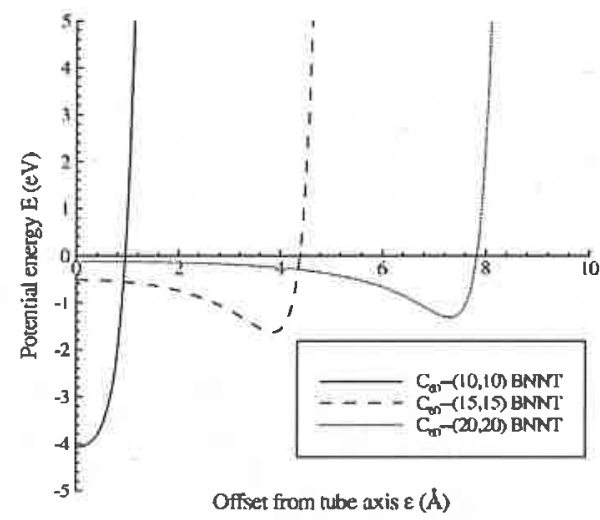

(b).

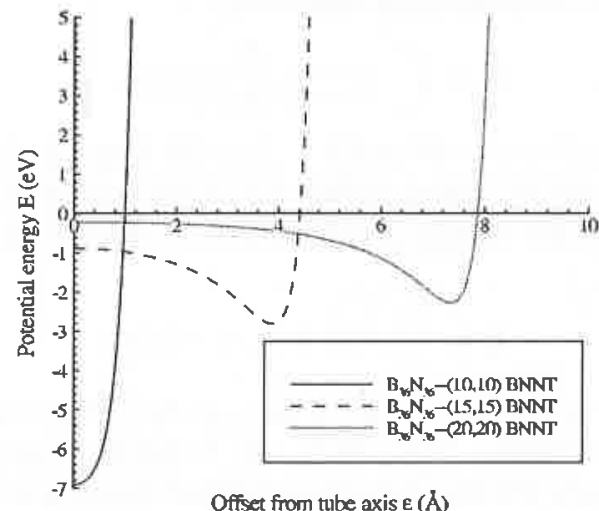

(d).

Figure 8. Potential energy of an offset fullerene inside a nanotube, with respect to the radial distance $\varepsilon$ from the tube axis: (a) $\mathrm{C}_{60}-$ carbon nanotube, (b) $\mathrm{C}_{60}-\mathrm{BN}$ nanotube, (c) $\mathrm{B}_{36} \mathrm{~N}_{36}$-carbon nanotube and (d) $\mathrm{B}_{36} \mathrm{~N}_{36}-\mathrm{BN}$ nanotube.

tube surface in all radial directions. Therefore, it is necessary for the surface of the fullerene to be at equal radial distance away from the nanotube surface, whereas for a larger tube (such as $(15,15)$ and $(20,20)$ nanotubes) the fullerene can be closer to one side of the tube, since the distance from the surface of the fullerene to the far side is greater than the van der Waals radius where the van der Waals force starts to operate.

\section{Oscillating fullerenes inside nanotubes}

Finally, we look at the oscillation of an inner molecule inside an outer nanotube. As mentioned previously, for certain nanotubes, the preferred position of the molecule is on the tube axis. Here, for simplicity, we only consider the case where the fullerene, upon acceptance inside the nanotube, then stays symmetrically situated on the $z$-axis inside the nanotube of length $2 L$ throughout the motion, as shown in figure 9.

Again in order to obtain a simple mathematical model, we assume for all of the interactions that the frictional effect is negligible, since this is the case for a carbon nanotube oscillating inside another [21]. Upon neglecting the frictional effect, the oscillatory motion of the fullerene 


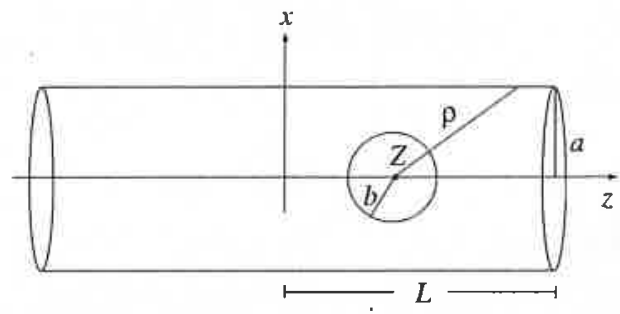

Figure 9. Oscillation of a fullerene inside a nanotube.

inside a nanotube can be readily explained using Newton's second law, namely

$$
M \frac{\mathrm{d}^{2} Z}{\mathrm{~d} t^{2}}=F(Z)
$$

where $M$ is the total mass of the fullerene, $Z(-L \leqslant Z \leqslant L)$ is the distance between the centre of the fullerene and the centre of the nanotube, and due to the symmetry of the problem we only need consider the force in the axial direction $F(Z)$. As shown in Cox et al [34], the total axial force $F(Z)$ for a $\mathrm{C}_{60}$ interacting with a carbon nanotube is given by

$$
F(Z)=2 \pi a \eta_{t}\left[P\left(r_{2}\right)-P\left(r_{1}\right)\right],
$$

where

$$
r_{1}=\sqrt{a^{2}+(Z+L)^{2}}, \quad r_{2}=\sqrt{a^{2}+(Z-L)^{2}},
$$

and the function $P(r)$ is defined by

$$
P(r)=\frac{2 \pi \eta_{f} b}{r}\left\{\frac{A}{4}\left(\frac{1}{(r+b)^{4}}-\frac{1}{(r-b)^{4}}\right)-\frac{B}{10}\left(\frac{1}{(r+b)^{10}}-\frac{1}{(r-b)^{10}}\right)\right\} .
$$

Again, we adapt these formulae and similar approaches to those of (4.2), (4.3) and (4.4) for $\mathrm{C}_{60}-$ $\mathrm{BN}$ nanotube, $\mathrm{B}_{36} \mathrm{~N}_{36}$-carbon nanotube and $\mathrm{B}_{36} \mathrm{~N}_{36}-\mathrm{BN}$ nanotube oscillations, respectively. We employ $(10,10)$ carbon and boron nitride nanotubes, and following [32] we assume that all nanotubes are of equal length, $2 L=54 \AA$. Upon plotting the total axial forces for each interaction type as shown in figure 10 , we find that our results are in excellent agreement with molecular dynamics simulations of Kang and Hwang [32], who predict the magnitude of $F_{v d W}$ at the tube extremities of $0.393 \mathrm{eV} \AA^{-1}$ for $\mathrm{C}_{60}-\mathrm{CNT}$ and $0.538 \mathrm{eV} \AA^{-1}$ for $\mathrm{C}_{60^{-}}$ BNNT. Further, we find that all forces admit similar behaviour such that it is very close to zero everywhere except at both the tube's extremities, where there is a peak-like force which ricochets the molecule towards the centre of the nanotube. This behaviour is also observed for the $\mathrm{C}_{60}$-carbon nanotube interaction in $\operatorname{Cox}$ et al [34], for which they observe that if $b<a \ll 2 L$ then effectively the total axial force behaves like two equal and opposite Dirac delta functions at the tube ends $Z=-L$ and $Z=L$. As such, we follow [34] by estimating $F(Z)$ by

$$
F(Z)=W[\delta(Z+L)-\delta(Z-L)],
$$

where $W$ is the pulse strength which is equivalent to the suction energy defined by (4.6). By substituting (6.2) into Newton's second law, the velocity of a fullerene can be determined as given by

$$
v=\left(2 W / M+v_{0}^{2}\right)^{1 / 2},
$$

noting that $\nu_{0}$ denotes the initial velocity, which in this paper is assumed to be zero. Equation (6.3) implies that the higher the pulse strength, the higher the velocity. From figure 10 , as the suction force is highest (giving rise to the highest pulse strength $W$ ), the highest velocity 


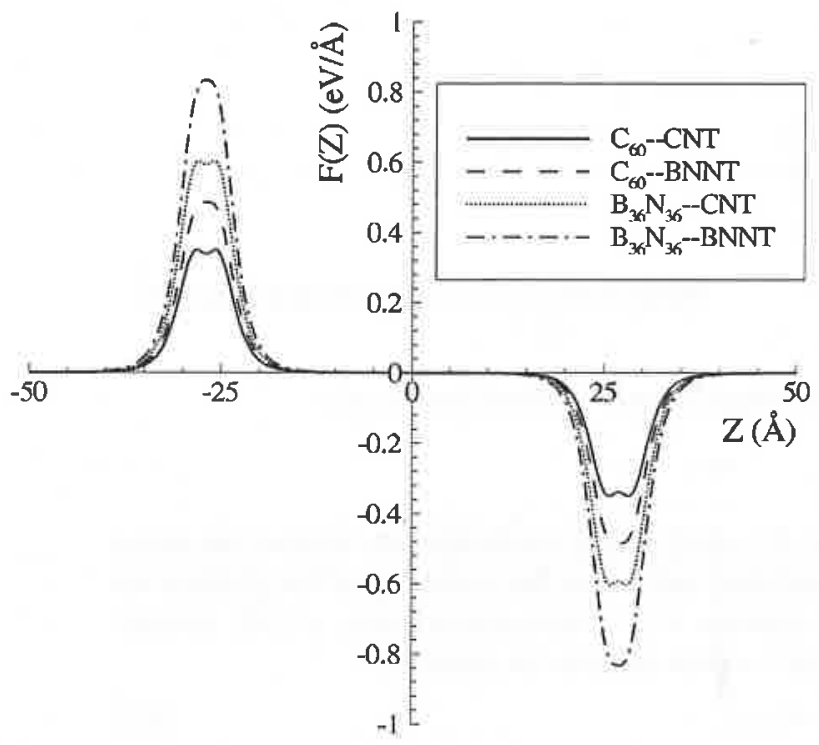

Figure 10. Total axial van der Waals force $F(Z)$ for oscillating fullerenes inside $(10,10)$ nanotubes.

Table 5. Velocity and frequency for each oscillator, assuming $(10,10)$ nanotubes of length $2 L=54 \AA$ and zero initial velocity.

\begin{tabular}{lllll}
\hline & \multicolumn{4}{c}{ Oscillators } \\
\cline { 2 - 5 } & $\mathrm{C}_{60}-\mathrm{CNT}$ & $\mathrm{C}_{60}-\mathrm{BNNT}$ & $\mathrm{B}_{36} \mathrm{~N}_{36}-\mathrm{CNT}$ & $\mathrm{B}_{36} \mathrm{~N}_{36}-\mathrm{BNNT}$ \\
\hline$v\left(\mathrm{~m} \mathrm{~s}^{-1}\right)$ & 907.857 & 1045.544 & 1064.095 & 1221.935 \\
$f(\mathrm{GHz})$ & 84.061 & 96.809 & 98.527 & 113.142 \\
\hline
\end{tabular}

is obtained for the fullerene $\mathrm{B}_{36} \mathrm{~N}_{36}$ travelling inside the $\mathrm{BN}$ nanotube. Further, (6.3) also implies that after being sucked in by a nanotube the fullerene will travel inside the tube at a constant velocity until it reaches the other open end, where there is a force which ricochets the molecule back towards the tube centre. Accordingly, the oscillation frequency can simply be determined from $f=v /(4 L)$. By using the data in table 1 , we may deduce that the velocity and frequency for each interaction type for the case of $2 L=54 \AA$ are as presented in table 5. We note that the frequency increases as the tube length decreases. While we predict the frequencies for $\mathrm{C}_{60}-\mathrm{CNT}^{\prime}$ and $\mathrm{C}_{60}-\mathrm{BNNT}$ oscillations to be 84.061 and $96.806 \mathrm{GHz}$ respectively, molecular dynamics simulations of Kang and Hwang [32] predict much lower frequencies, 45 and $60 \mathrm{GHz}$, respectively. The cause of this difference may be the fact that our model assumes a frictionless environment and also that the predicted frequencies of Kang and Hwang [32] are obtained at a certain time after the suction of the fullerene begins. However, the velocity predicted here for a $\mathrm{C}_{60}$ travelling inside a $(10,10)$ carbon nanotube is in reasonable agreement with molecular dynamics simulations of Qian et al [39], who predict $840 \mathrm{~m} \mathrm{~s}^{-1}$.

\section{Conclusions}

In summary, we extend the analytical mathematical expressions of Girifalco [35] and Cox et al $[33,34]$ to study the molecular interactions of boron nitride and carbon nanostructures. It is shown here that this simple classical applied mathematical model can be used to capture the major underlying mechanisms of the encapsulations of a fullerene through an open end 
of a nanotube. Our results show that the interaction between $\mathrm{B}_{36} \mathrm{~N}_{36}-\mathrm{B}_{36} \mathrm{~N}_{36}$ is stronger than $\mathrm{C}_{60}-\mathrm{C}_{60}$ and $\mathrm{C}_{60}-\mathrm{B}_{36} \mathrm{~N}_{36}$ since it admits the lowest minimum energy. For the interactions of a fullerene and a nanotube, we again find that the interaction of a molecule with a boron nitride nanostructure is more energetically favourable than the interaction of purely carbon molecules. In particular, this paper presents the minimum radii required for a nanotube that will accept a fullerene from rest. For $\mathrm{C}_{60}$-carbon nanotube, $\mathrm{C}_{60}-\mathrm{BN}$ nanotube, $\mathrm{B}_{36} \mathrm{~N}_{36}$-carbon nanotube and $\mathrm{B}_{36} \mathrm{~N}_{36}-\mathrm{BN}$ nanotube, these minimum radii are given by $6.277,6.293,6.243$ and $6.258 \AA$, respectively. This paper confirms that inside a carbon nanotube a fullerene tends to have a preferred location at an inter-atomic distance apart from the inner tube surface, where the molecule has its minimum energy. Inside $(10,10)$ nanotubes (CNNT and BNNT), fullerenes $\left(\mathrm{C}_{60}\right.$ and $\left.\mathrm{B}_{36} \mathrm{~N}_{36}\right)$ prefer to be at the centre of the cross-section of the nanotube. As the tube becomes larger, the molecule inside tends to be closer to one side of the nanotube wall. Further, the suction energy, which can be equated directly to the kinetic energy of the fullerene, is studied for each interaction. Our results show that the highest velocity is obtained from the fullerene $\mathrm{B}_{36} \mathrm{~N}_{36}$ inside a $\mathrm{BN}$ nanotube. The second and third fastest configurations are $\mathrm{C}_{60}-\mathrm{BN}$ nanotube and $\mathrm{B}_{36} \mathrm{~N}_{36}$-carbon nanotube respectively, and $\mathrm{C}_{60}$-carbon nanotube is the slowest. The oscillation frequency is also investigated here assuming that the centre of the fullerene moves along the tube axis, which is assumed in order to keep the model simple. In fact, this situation occurs for the case of a fullerene (either $\mathrm{C}_{60}$ or $\mathrm{B}_{36} \mathrm{~N}_{36}$ ) moving inside a $(10,10)$ nanotube (either carbon or boron nitride nanotubes). Without a frictional effect, the oscillating $\mathrm{B}_{36} \mathrm{~N}_{36}$ inside a $\mathrm{BN}$ nanotube gives rise to the highest frequency. To obtain a more realistic model, future work is needed to incorporate the frictional effect or to account for the energy dissipation between a fullerene and a nanotube.

\section{Acknowledgments}

The authors are grateful to the Australian Research Council for support through the Discovery Project Scheme and the provision of an Australian Postdoctoral Fellowship for NT and an Australian Professorial Fellowship for JMH. The authors also wish to acknowledge Barry Cox for many helpful comments and discussions.

\section{References}

[1] Zettl A 1996 Non-carbon nanotubes Adv. Mater. 8 443-5

[2] Ishigami M, Aloni S and Zettl A 2003 Properties of Boron Nitride Nanotubes in Scanning Tunneling Microscopy/Spectroscopy and Related Techniques: 12th Int. Conf. ed P M Koenraad and M Kemerink (New York: American Institute of Physics) pp 94-99 (http://www.physics.berkeley.edu/research/zettl/pdf/ 289.AIP696ishigami.pdf)

[3] Mickelson W, Aloni A, Han W-Q, Cumings J and Zettl A 2003 Packing $\mathrm{C}_{60}$ in boron nitride nanotubes Science $300467-9$

[4] Hirano T, Oku T and Suganuma K 2000 Atomic structure and electronic state of boron nitride fullerenes and nanotubes Diamond Relat. Mater. 9 625-8

[5] Vavro J, Llaguno M C, Satishkumar B C, Luzzi D E and Fischer J E 2002 Electrical and thermal properties of $\mathrm{C}_{60}$-filled single-wall carbon nanotubes Appl. Phys. Lett. $801450-2$

[6] Noya E G, Srivastava D, Chemozatonskii L A and Menon M 2004 Themal conductivity of carbon nanotube peapods Phys. Rev. B 70115416

[7] Okada S, Saito S and Oshiyama A 2001 Energetics and electronic structures of encapsulated $C_{60}$ in a carbon nanotube Phys. Rev. Lett. $863835-8$

[8] Zettl A, Cumings J, Han W-Q and Mickelson W 2002 Boron Nitride Nanotube Peapods in Structural and Electmnic Properties of Molecular Nanostructures ed H Kuzmany et al (New York: American Institute of Physics) pp 140-144 (http://www.physics.berkeley.edu/research/zettl/pdf/276.AIP633zettl.pdf)

[9] Hodak M and Girifalco L A 2003 Ordered phases of fullerene molecules formed inside carbon nanotubes Phys. Rev. B 67075419 
[10] Hodak M and Girifalco L A 2003 Systems of $C_{60}$ moleucles inside $(10,10)$ and $(15,15)$ nanotube: a Mote Carlo study Phys. Rev. B 68085405

[11] Trave A, Ribeiro F J, Louie S G and Cohen M L 2004 Energetics and structural characterization of $\mathrm{C}_{60}$ polymerization in BN and carbon nanopeapods Phys. Rev. B 70205418

[12] Okada S, Saito $S$ and Oshiyama A 2001 Semiconducting form of the first-row elements: $\mathrm{C}_{60}$ chain encapsulated in BN nanotubes Phys. Rev. B 64201303 (R)

[13] Ulbricht $\mathrm{H}$ and Hertel T 2003 Dynamics of $\mathrm{C}_{60}$ encapsulation into single-walled carbon nanotubes $J$. Phys. Chem. B 107 14185-90

[14] Ulbricht H, Moos G and Hertel T 2003 Interaction of $\mathrm{C}_{60}$ with carbon nanotubes and graphite Phys. Rev. Lett. 90095501

[15] Berber S, Kwon Y K and Tomanek D 2002 Microscopic formation mechanism of nanotube peapods Phys. Rev. Letl. 88185502

[16] Zope R R, Baruah T, Pederson M R and Dunlap B I 2005 Theoretical infared, Raman, and optical spectra of the $\mathrm{B}_{36} \mathrm{~N}_{36}$ cage Phys. Rev. A 71025201

[17] Batsta R J C, Mazzoni M S C and Chacham H 2007 Boron nitride fullerene B36N36 doped with transition metal atoms: first-principles calculations Phys. Rev. B 75035417

[18] Alexandre S S, Mazzoni M S C and Chacham H 1999 Stability, geometry, and electronic structure of the boron nitride B36N36 fullerene Appl. Phys. Lett. 75 61-3

[19] Oku T, Hirano T, Kuno M, Kusunose T, Niihara K and Suganuma K 2000 Synthesis, atomic strcutures and properties of carbon and boron nitride fullerene materials Mater. Sci. Eng. B 74 206-17

[20] Oku T, Narita I and Nishiwaki A 2004 Synthesis, atomic strcutures and electronic states of boron nitride nanocage clusters and nanotubes Mater. Manuf. Process. 19 1215-39

[21] Cumings J and Zettl A 2000 Low-friction nanoscale linear bearing realized from multiwalled carbon nanotubes Science $289602-4$

[22] Yu M F, Yakobson B I and Ruoff R S 2000 Controlled sliding and pullout of nested shells in individual multiwalled carbon nanotubes J. Phys. Chem. B 104 8764-7

[23] Legoas S B, Coluci V R, Braga S F, Coura P Z, Dantas S O and Galväo D S 2003 Molceular-dynamics simulations of carbon nanotubes as gigahertz oscillators Phys. Rev. Lett. 90055504

[24] Zheng Q and Jiang Q 2002 Multiwalled carbon nanotubes as gigahertz oscillators Phys. Rev. Lett. 88045503

[25] Zheng Q, Liu J Z and Jiang Q 2002 Excess van der Waals interaction energy of a multiwalled carbon nanotube with an extruded core and the induced core oscillation Phys. Rev. B 65245409

[26] Rivera J L, McCabe C and Cumming P T 2003 Oscillatory behavior of double-walled nanotubes under extension: a simple nanoscale damped spring Nano Left. 3 1001-5

[27] Rivera J L, McCabe C and Cumming P T 2005 The oscillatory damped behavior of incommensurate doublewalled carbon nanotubes Nanotechnology 16 186-98

[28] Liu P, Zhang Y W and Lu C 2005 Oscillatory behavior of C60-nanotube oscillators: a molecular-dynamics study J. Appl. Phys. 97094313

[29] Hilder T A and Hill J M 2007 Continuous versus discrete for interacting carbon nanostructures J. Phys. A. Math. Theor, 40 3851-68

[30] Verberck $\mathrm{B}$ and Michel K H 2006 Nanotube field of $\mathrm{C}_{60}$ molecules in carbon nanotubes: atomistic versus continuous approach Phys. Rev. B 74045421

[31] Lee J H 2006 A study on a boron-nitride nanotube as a gigahertz oscillator J. Korean Phys. Soc. 49 172-6

[32] Kang $J$ W and Hwang $\mathrm{H} J 2004$ Comparison of $\mathrm{C}_{60}$ encapsulations into carbon and boron nitride nanotubes J. Phys.: Condens. Matter $163901-8$

[33] Cox B J, Thamwattana N and Hill J M 2007 Mechanics of atoms and fullerenes in single-walled carbon namotubes. I. Acceptance and suction energies Proc. R. Soc. A 463 461-76

[34] Cox B J, Thamwattana N and Hill J M 2007 Mechanics of atoms and fullerenes in single-walled carbon nanotubes. II. Oscillatory behaviour Proc. R. Soc. A 463 477-94

[35] Girifalco L A 1992 Molecular properties of $\mathrm{C}_{60}$ in the gas and solid phases J. Phys. Chem. $96858-61$

[36] Ruoff R S and Hickman A P 1993 van der Waals binding of fulletenes to a graphite plane J. Phys. Chem. $972494-6$

[37] Iglesias-Groth S, Breton J and Girardet C 1997 An analytical approach for interlayer interaction in spherical buckyonions Chem. Phys. Lett. 264 351-8

[38] Girifalco L A, Hodak M and Lee R S 2000 Carbon nanotubes, buckyballs, ropes, and a universal graphitic potential Phys. Rev. B $6213104-10$

[39] Qian D, Liu W K and Ruoff R S 2001 Mechanics of $\mathrm{C}_{60}$ in nanotubes J. Phys. Chem. B 105 10753-8

[40] Hodak M and Girifalco L A 2001 Fullerenes inside carbon nanotubes and multi-walled carbon nanotubes: optimum and maximum sizes Chem. Phys. Lett. 350 405-11 